\title{
Sensor Fusion Using Fuzzy Logic EnHANCED Kalman Filter for Autonomous Vehicle Guidance in Citrus Groves
}

\author{
V. Subramanian, T. F. Burks, W. E. Dixon
}

\begin{abstract}
This article discusses the development of a sensor fusion system for guiding an autonomous vehicle through citrus grove alleyways. The sensor system for path finding consists of machine vision and laser radar. An inertial measurement unit (IMU) is used for detecting the tilt of the vehicle, and a speed sensor is used to find the travel speed. A fuzzy logic enhanced Kalman filter was developed to fuse the information from machine vision, laser radar, IMU, and speed sensor. The fused information is used to guide a vehicle. The algorithm was simulated and then implemented on a tractor guidance system. The guidance system's ability to navigate the vehicle at the middle of the path was first tested in a test path. Average errors of $1.9 \mathrm{~cm}$ at $3.1 \mathrm{~m} \mathrm{~s}^{-1}$ and $1.5 \mathrm{~cm}$ at $1.8 \mathrm{~m} \mathrm{~s}^{-1}$ were observed in the tests. A comparison was made between guiding the vehicle using the sensors independently and using fusion. Guidance based on sensor fusion was found to be more accurate than guidance using independent sensors. The guidance system was then tested in citrus grove alleyways, and average errors of $7.6 \mathrm{~cm}$ at $3.1 \mathrm{~m} \mathrm{~s}^{-1}$ and $9.1 \mathrm{~cm}$ at $1.8 \mathrm{~m} \mathrm{~s}^{-1}$ were observed. Visually, the navigation in the citrus grove alleyway was as good as human driving.
\end{abstract}

Keywords. Autonomous vehicle, Fuzzy logic, Guidance, Kalman filter, Sensor fusion, Vision.

$\mathrm{T}$ here is a current need in the Florida citrus industry to automate citrus grove operations. This need is due to reduction in the availability of labor, rising labor cost, and potential immigration challenges. Autonomous vehicles would be an important part of an automated citrus grove. Autonomous vehicles can operate accurately for a longer duration of operation than when using a human driver. In a scenario where an automated citrus harvester is used, the operator can monitor both the harvester and the vehicle instead of being solely the driver. Based on citrus groves identified for testing the autonomous navigation of the tractor used by Subramanian et al. (2006), it was estimated that the ability to navigate in the middle of the identified citrus alleyways with an accuracy of $15 \mathrm{~cm}$ deviation from the center would be acceptable. In general, this estimate is dependent on the size of the vehicle, the alleyway width, layout of the citrus groves, and the application for which the autonomous vehicle is used. Numerous autonomous vehicles for agricultural applications have been described in the literature. The guidance systems developed by Noguchi et al. (2002) and Chen et al. (2003) were for operation in paddy fields for transplanting rice, whereas the guidance system de-

Submitted for review in December 2007 as manuscript number PM 7305; approved for publication by the Power \& Machinery Division of ASABE in July 2009.

The authors are Vijay Subramanian, Graduate Student, Department of Agricultural and Biological Engineering, Thomas F. Burks, ASABE Member Engineer, Associate Professor, Department of Agricultural and Biological Engineering, and Warren E. Dixon, Assistant Professor, Department of Mechanical and Aerospace Engineering, University of Florida, Gainesville, Florida. Corresponding author: Vijay Subramanian, Department of Agricultural and Biological Engineering, P.O. Box 110570; University of Florida, Gainesville, FL 32611; phone: 352-392-1864; fax: 352-392-8476; e-mail: vijays@ufl.edu. veloped by Nagasaka et al. (2004) was tested in soybean fields. Satow et al. (2004) developed a laser based crop row sensor for navigating a tractor through crop rows.

Machine vision is a useful sensing methodology for guidance. Its long range aids in faster decision making before a hard turn is to be performed or for faster obstacle detection and is quite robust to minor variations in the path boundaries. However, it lacks the centimeter-level accuracy of laser radar in detecting the distance to close range path boundaries or obstacles. The accuracy of ladar is useful in keeping the vehicle accurately positioned in the path. However, this accuracy makes the information noisy if there are minor variations in the path boundaries. The development of an autonomous vehicle guidance system for citrus grove navigation using machine vision and laser radar (ladar) based guidance systems was discussed by Subramanian et al. (2006). Both machine vision and ladar based guidance performed with a maximum error of $6.1 \mathrm{~cm}$ in test paths. The machine vision based guidance system had also guided a tractor through an alleyway of a citrus grove, keeping the vehicle visually in the center of the path with no collisions with the trees. It was found that ladar based guidance was more accurate than vision based guidance in well defined paths within a speed of $3.1 \mathrm{~m} \mathrm{~s}^{-1}$, whereas the vision based guidance was able to keep the vehicle in the middle of the path in several types of paths with less accuracy. In the approach taken in previous research, the uneven tree canopy in citrus grove alleyways posed problems for guidance when using vision and ladar separately (Subramanian et al., 2006). With a vision based guidance configuration using a forward-facing camera, branches closer to the vehicle than the camera's field of view are not accounted for in the vision based guidance, and navigating near the end of the row is not feasible as the last trees go out of the field of view of the camera. Ladar, with a wider field of view and closer 
range, is useful for guidance in these situations. However, in a given alleyway, it is not uncommon to find a few trees absent. In such cases, ladar based guidance gives erroneous paths. Variations in foliage over a short distance also posed problems in using ladar for guidance. Fusing the complementary information from these sensors, namely the long-range information from vision and the short-range accuracy of ladar, would be beneficial.

This article presents the research further undertaken to fuse the information from these sensors to make the composite information from them more robust and make the overall guidance system more reliable. The development of the Kalman filter for fusion is first discussed, followed by a description of the fuzzy logic system to augment the filter. Finally, the experiments conducted to test the performance of the fused guidance system are described.

\section{Approach}

Several methods for fusion have been reported in the literature. These include, but are not limited to, neural networks (Davis and Stentz, 1995; Rasmussen, 2002), variations of Kalman filter (Paul and Wan, 2005), statistical methods (Wu et al., 2002), behavior based methods (Gage and Murphy, 2000), voting, fuzzy logic (Runkler et al., 1998), and combinations of these (Mobus and Kolbe, 2004). The process of fusion might be performed at various levels ranging from sensor level to decision level (Klein, 1999).

In our research, the tree canopy causes the sensor measurements to be fairly noisy. Therefore, the choice of method was narrowed down to one that could help in reducing the noise and also aid in fusing the information from the sensors. Methods such as neural networks, behavior based methods, fuzzy logic, and voting are not widely used primarily for reducing noise. Kalman filtering is a widely used method for eliminating noisy measurements from sensor data and also for sensor fusion. Kalman filter can be considered as a subset of statistical methods because of the use of statistical models for noise. Paul and Wan (2005) used two Kalman filters for accurate state estimation and for terrain mapping for navigating a vehicle through unknown environments. The state estimation process fused the information from three onboard sensors to estimate the vehicle location. Simulated results showed the feasibility of the method. Han et al. (2002) used a Kalman filter to filter DGPS (Differential Global Positioning System) data for improving positioning accuracy for parallel tracking applications. The Kalman filter smoothed the data and reduced the cross-tracking error. Based on the good results obtained in the previous research for fusion and noise reduction, a Kalman filter was chosen for our research as the method to perform the fusion and filter the noise in sensor measurements.

The use of a Kalman filter with fixed parameters has drawbacks. Divergence of the estimates, wherein the filter continually tries to fit a wrong process, is a problem that is sometimes encountered with a Kalman filter. Divergence may be attributed to system modeling errors, noise variances, ignored bias, and computational rounding errors (Fitzgerald, 1971). Another problem in using a simple Kalman filter is that the reliability of the information from the sensors depends on the type of path in the grove. For example, if there is a tree missing on either side of the path while the vehicle is navigating through a grove, the information from the ladar is no longer useful and the guidance system has to rely on the information from vision alone. Therefore, if a white-noise model is assumed for the process and measurements, the reliability factor of a sensor in the Kalman filter has to be constantly updated. Abdelnour et al. (1993) used fuzzy logic in detecting and correcting the divergence. Sasladek and Wang (1999) used fuzzy logic with an extended Kalman filter to tackle the problem of divergence for an autonomous ground vehicle. The extended Kalman filter reduced the position and velocity error when the filter diverged. The use of fuzzy logic also allowed a lower-order state model to be used. For our research, fuzzy logic is used in addition to Kalman filtering to overcome divergence and to update the reliability parameter in the filter. Sasladek and Wang (1999) used fuzzy logic to reduce divergence in the Kalman filter, whereas in this research, apart from using fuzzy logic to reduce divergence, fuzzy logic is also used to update the usability of sensors in different environmental conditions, as discussed in a later section. Whereas Sasladek and Wang (1999) present simulation results of their method, this research implements the methods on a real-world system for citrus grove navigation application and presents the performance.

The main objective of this research was to fuse the information from machine vision and laser radar using a fuzzy logic enhanced Kalman filter to make the autonomous vehicle guidance system more reliable in a citrus grove than when using the sensors individually. The following specific objectives were chosen:

- Develop a fuzzy logic enhanced Kalman filter fusion method.

- Confirm operation of the method by simulation.

- Implement the method on a tractor fitted with the required sensors.

- Test the navigation performance on a custom-designed test track.

- Test the navigation performance in citrus grove alleyways.

\section{Materials ANd Methods}

The guidance system developed by Subramanian et al. (2006) consisted of machine vision and laser radar as the main guidance sensors. The camera used for machine vision was a Sony FCB-EX780S "block" single CCD analog color video camera with a standard lens and resolution of $640 \times$ 480. The ladar used was the Sick LMS 200 (Sick, Inc., Minneapolis, Minn.), which has a maximum sweep angle of $180^{\circ}$ and maximum range of $80 \mathrm{~m}$. The ladar was operated at $1 \mathrm{~mm}$ accuracy with a range of $8 \mathrm{~m}$. A PC processed the information from the sensors and sent the error to a microcontroller. The microcontroller controlled the steering using a PID control system. An encoder fed back steering angle measurements to the microcontroller. At present, a tri-axial inertial measurement unit (IMU) (3DM, MicroStrain, Inc., Williston, Vt.) and an ultrasonic speed sensor (Trak-Star, Micro-Trak Systems, Inc., Eagle Lake, Minn.) have been added to the existing system to aid the guidance and sensor fusion. The IMU provided the vehicle heading, and the speed sensor provided the vehicle speed. These helped in estimating the error using the Kalman filter. Communication with both of these sensors was through RS-232 serial communication. The IMU was 


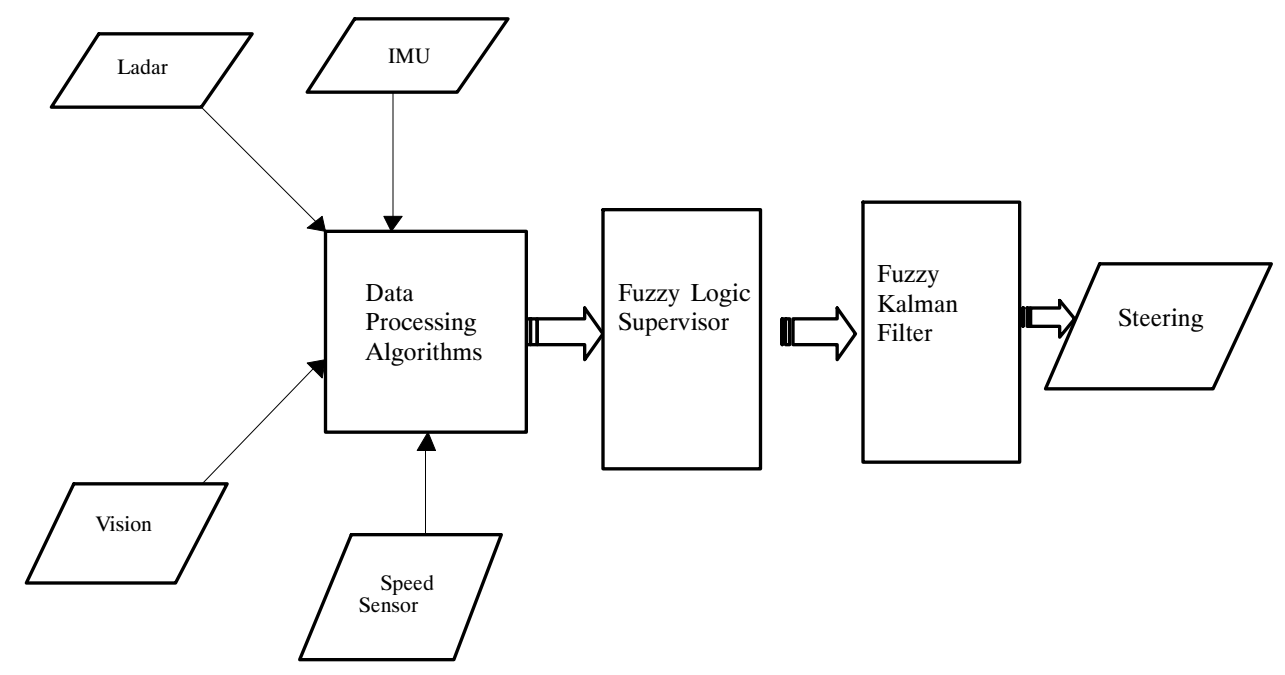

Figure 1. Fusion-based guidance system architecture.

mounted inside the tractor cabin on the roof just below the ladar and camera. The speed sensor was mounted below the tractor. A special serial RS-422 communication port was added to the PC to enable a ladar refresh rate of $30 \mathrm{~Hz}$. The architecture of the major components involved in the fusion based guidance system is shown in figure 1 .

The vision and ladar algorithms are described in detail by Subramanian et al. (2006). The vision algorithm used colorbased segmentation of trees and ground, followed by morphological operations to clean the segmented image. The path center was determined as the center between the tree boundaries. The error was calculated as the difference between the center of the image and the path center identified in the image. The error was converted to real-world distance using prior pixel to distance calibration. To calculate the required heading of the vehicle, a line was fit for the path center in the image representing the entire alleyway. The angle between this line and the image center line was determined as the required heading for the vehicle to navigate the alleyway with low lateral error. The ladar algorithm used a distance threshold to differentiate objects from the ground. An object of a minimum width was identified as a tree. The midpoint between the trees identified on either side was determined as the path center. The errors measured using vision and ladar were adjusted for tilt using the tilt information from the IMU. The information from the sensors was used in the fusion algorithm, and the resulting error in lateral position was passed to a PID control system, which controlled the steering angle to reduce the error to zero.

\section{KALMAN FiLter}

A Kalman filter was used to fuse the information from the vision, ladar, IMU, and speed sensors. The models and implementation equations for the Kalman filter (Zarchan and Musoff, 2005) are described below.

\section{State Transition Model}

The state transition model predicts the coordinates of the state vector $\boldsymbol{x}$ at time $k+1$ based on information available at time $k$ and is given by:

$$
\boldsymbol{x}(k+1)=\boldsymbol{A x}(k)+\boldsymbol{w}(k)
$$

where

$$
\begin{aligned}
& k=\text { time instant } \\
& \boldsymbol{w}=\text { process noise, which was assumed to be Gaussian } \\
& \text { with zero mean. } \\
& \qquad \boldsymbol{x}(k)=\left[d(k) \theta(k) \theta_{R}(k) v(k)\right]^{T}
\end{aligned}
$$

where

$$
\begin{aligned}
d(k) \in R= & \text { lateral position error of the vehicle in the } \\
& \text { path, which is the difference between the } \\
& \text { desired lateral position and the actual lateral } \\
& \text { position } \\
\theta(k) \in R= & \text { current vehicle heading } \\
\theta_{R}(k) \in R= & \text { required vehicle heading } \\
v(k) \in R= & \text { vehicle linear velocity. }
\end{aligned}
$$

It is to be noted that only the change in heading was used for estimating the position error, which affects the overall guidance. The absolute heading was included in the filter to remove noise from the IMU measurements. Figure 2 shows the vehicle in the path with the state vector variables.

The state transition matrix, $A(k) \in R^{4 \times 4}$ is defined as:

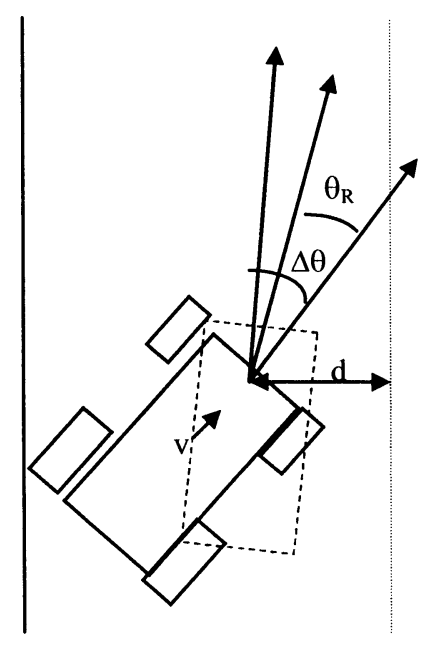

Figure 2. Vehicle in the path with the state vector variables. 


$$
\boldsymbol{A}=\left[\begin{array}{cccc}
1 & 0 & 0 & t^{*} \sin \theta \\
0 & 1 & 0 & 0 \\
0 & 0 & 1 & 0 \\
0 & 0 & 0 & 1
\end{array}\right]
$$

where

$t \quad=$ time step

$\Delta \theta=$ change in heading; this value was calculated from the filtered IMU measurements for each time step $k$.

The use of a variable sensor input such as $\Delta \theta$ in the state transition matrix is consistent with the method described by Kobayashi et al. (1998). The state estimate error covariance matrix, $\boldsymbol{P} \in R^{4 \times 4}$, gives a measure of the estimated accuracy of the state estimate $\boldsymbol{x}(k+1)$.

$$
\boldsymbol{P}(k+1)=\boldsymbol{A P}(k) \boldsymbol{A}^{T}+\boldsymbol{Q}(k)
$$

where $Q \in R^{4 \times 4}$ denotes the covariance matrix for process noise $\boldsymbol{w}$.

The estimation process is difficult to directly observe; hence, the process noise covariance is uncertain. Therefore, the initial variance values, which are the diagonal elements in the matrix, were assumed as follows:

- Variance for process position noise $=2 \mathrm{~cm}$.

- Variance for process current heading noise $=0.01^{\circ}$.

- Variance for required heading noise $=0.01^{\circ}$.

- Variance for process velocity noise $=10^{-4} \mathrm{~m} \mathrm{~s}^{-1}$.

These values were intuitively assumed to be the amount of noise variance that the process could add. As discussed further in this article, the process noise covariance matrix, $\boldsymbol{Q}$, was updated at each step of the operation of the Kalman filter. Therefore, the initial assumptions of the matrix values do not significantly affect the overall guidance of the vehicle. These sensor measurements were assumed to be independent of the other sensors. This was done for simplicity. Later it was observed from simulations and experiments that the guidance of the vehicle, with this assumption, was accurate enough to keep the vehicle close to the alleyway center. Hence, the covariance values, which are the off-diagonal elements, are zero:

$$
\begin{aligned}
\boldsymbol{Q} & =\left[\begin{array}{cccc}
q_{X} & 0 & 0 & 0 \\
0 & q_{\theta_{C}} & 0 & 0 \\
0 & 0 & q_{\theta_{R}} & 0 \\
0 & 0 & 0 & q_{V}
\end{array}\right] \\
& =\left[\begin{array}{cccc}
2 & 0 & 0 & 0 \\
0 & 0.01 & 0 & 0 \\
0 & 0 & 0.01 & 0 \\
0 & 0 & 0 & 10^{-4}
\end{array}\right]
\end{aligned}
$$

\section{Measurement Model}

This model defines the relationship between the state vector, $\boldsymbol{x}$, and the measurements processed by the filter, $z$ :

$$
\begin{gathered}
z(k)=\boldsymbol{H} \boldsymbol{x}(k)+\boldsymbol{u}(k) \\
z(k)=\left[x_{C}(k) x_{L}(k) \theta_{C}(k) \theta_{\mathrm{IMU}}(k) v(k)\right]^{\mathrm{T}}
\end{gathered}
$$

where $z(k) \in R^{5}$ denotes a state vector composed of measured values of:
- Lateral position error of the vehicle in the path from vision algorithm, denoted by $x_{C}(k) \in R$.

- Lateral position error of the vehicle in the path from ladar algorithm, denoted by $x_{L}(k) \in R$.

- Required heading of the vehicle determined from vision algorithm, denoted by $\theta_{C}(k) \in R$.

- Current heading of the vehicle measured using IMU, denoted by $\theta_{\mathrm{IMU}}(k) \in R$.

- Vehicle linear velocity measured using the speed sensor, denoted by $v(k) \in R$.

The observation matrix, $\boldsymbol{H}(k)$ is defined as:

$$
\boldsymbol{H}=\left[\begin{array}{lllll}
1 & 1 & 0 & 0 & 0 \\
0 & 0 & 1 & 0 & 0 \\
0 & 0 & 0 & 1 & 0 \\
0 & 0 & 0 & 0 & 1
\end{array}\right]^{T}
$$

The observation matrix relates the state vector, $\boldsymbol{x}$, with the measurement vector, $\boldsymbol{z}$. The first two rows of $\boldsymbol{H}$ relate the lateral position errors from vision and ladar, the third and fourth rows relate the angles from vision and IMU, and the last row relates the velocity. The measurement noise, denoted by $\boldsymbol{u}(k)$, is assumed to be Gaussian with zero mean and standard deviations estimated experimentally with the different sensors.

\section{FILTER GAIN}

The filter gain, $\boldsymbol{G}$, is the factor used to minimize the posteriori error covariance, $\boldsymbol{P}$ :

$$
\boldsymbol{G}(k)=\boldsymbol{P}(k+1) \boldsymbol{H}^{T}\left(\boldsymbol{H} \boldsymbol{P}(k+1) \boldsymbol{H}^{T}+\boldsymbol{R}\right)^{-1}
$$

where $\boldsymbol{R}$ is the measurement noise covariance matrix.

To determine $\boldsymbol{R}$, the vehicle was set stationary in the middle of the path, each sensor (except the speed sensor) mounted on the vehicle was turned on independently, and the information from the sensors was collected for $30 \mathrm{~s}$. For the camera, the vision algorithm (Subramanian et al., 2006) was run for $30 \mathrm{~s}$ and the path error and the heading angle determined were recorded. For the ladar, the ladar path segmenting algorithm (Subramanian et al., 2006) was run for $30 \mathrm{~s}$ and the path error measurements were recorded. For the IMU, the sensor was run for $30 \mathrm{~s}$ and the heading angle measured by the sensor was recorded. For the speed sensor, the vehicle was run at three different constant speeds and the speed sensor measurement was collected for $30 \mathrm{~s}$. The measurement noise covariance matrix, denoted by $\boldsymbol{R}$, was determined from these recorded measurements and was found to be:

$$
\begin{aligned}
\boldsymbol{R} & =\left[\begin{array}{ccccc}
\sigma_{X c} & 0 & 0 & 0 & 0 \\
0 & \sigma_{X l} & 0 & 0 & 0 \\
0 & 0 & \sigma_{\theta c} & 0 & 0 \\
0 & 0 & 0 & \sigma_{\theta i m u} & 0 \\
0 & 0 & 0 & 0 & \sigma_{V}
\end{array}\right] \\
& =\left[\begin{array}{ccccc}
1.07 & 0 & 0 & 0 & 0 \\
0 & 0.15 & 0 & 0 & 0 \\
0 & 0 & 0.0017 & 0 & 0 \\
0 & 0 & 0 & 0.0001 & 0 \\
0 & 0 & 0 & 0 & 0
\end{array}\right]
\end{aligned}
$$




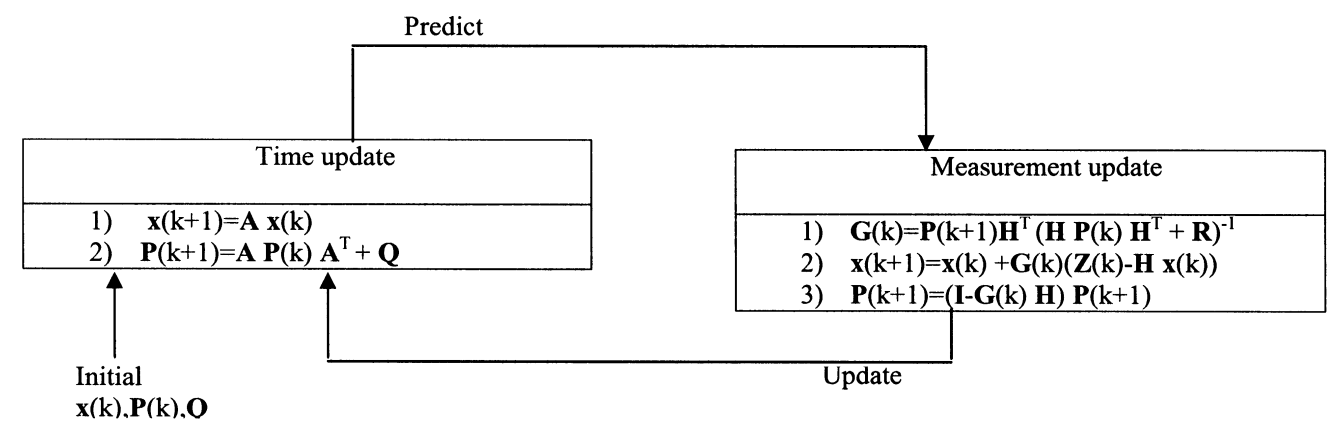

Figure 3. Kalman filter operation.

where

$\sigma_{X c}=$ variance of the error in the lateral position of the vehicle, as determined from vision algorithm measurements

$X l \quad=$ variance of the error in the lateral position of the vehicle, as determined from ladar algorithm measurements

$\sigma_{\theta c}=$ variance of the error in heading, as determined from the vision algorithm

$\sigma_{\theta \mathrm{i} m u}=$ variance of the error in heading, as determined from IMU measurement

$\sigma_{V}=$ variance of the error in velocity, as determined from speed sensor measurements.

The variables $x_{C}$ and $\theta_{C}$ were measurements from the same camera; however, the vision algorithm used different regions of the image to estimate them. The variables $x_{C}, x_{L}$, $\theta_{\mathrm{IMU}}$, and $v$ were measurements from different sensors operating independent of each other. These measurements were assumed to be statistically independent. Hence, the covariance values are zero. The variance of the velocity measurements was zero. This can be attributed to the low resolution $\left(0.5 \mathrm{~m} \mathrm{~s}^{-1}\right)$ of the speed sensor.

Figure 3 shows the operation of the Kalman filter. The filter estimates the process state $\boldsymbol{x}$ at some time $k+1$ and estimates the covariance $\boldsymbol{P}$ of the error in the estimate. The filter then obtains the feedback from the measurement $z$. Using the filter gain $\boldsymbol{G}$ and the measurement $\boldsymbol{z}$, it updates the state $\boldsymbol{x}$ and the error covariance $\boldsymbol{P}$. This process is repeated as new measurements come in and the error in estimation is continuously reduced.

\section{Reliability Factor of Primary Guidance Sensors in THE KaLMAN Filter}

As discussed previously, ladar is accurate at short range for the given mounting arrangement, and machine vision is good at providing the overall heading of the vehicle. By experimentation in a citrus grove, the following observations were made: tree foliage is highly variable, trees can be absent on either or both sides of the path, and some trees can be small enough for ladar to not recognize them as trees. In such a variable path condition, it is not feasible to have constant noise values for the ladar and machine vision. For example, when the ladar does not recognize trees on either side, the guidance becomes more reliable if the process noise for the ladar is increased to a high value such that vision takes over as the only guidance sensor. When the vehicle gets to the end of a tree row, vision is no longer useful, so ladar can be made the sole guidance sensor to cross the last tree. A fuzzy logic system was used to decide the reliability of the sensor. The reliability was changed in the Kalman filter by changing the measurement noise covariance matrix $\boldsymbol{R}$. This is discussed in the following section.

\section{Fuzzy Logic Sensor Supervisor}

A fuzzy logic based supervisor was used to decide which sensor is more reliable at different locations in the grove alleyway. The fuzzy logic algorithm was implemented in software using $\mathrm{C}++$ in the PC. The input to the fuzzy logic supervisor was the horizontal distance of the vehicle centerline from the trees on either side of the vehicle. Both vision and ladar input their corresponding distance values. Altogether, there are four input values: vision left tree distance, vision right tree distance, ladar left tree distance, and ladar right tree distance. These inputs were divided into three linguistic variables: reasonable, unreasonable, and zero (fig. 4).

A triangle-based fuzzification method was used to fuzzify the input values. The input membership function relates the input $x$ to the linguistic variables reasonable, unreasonable, and zero (fig. 4b). The meaning of the linguistic variables is literally whether the distance from the tree row is reasonable or not. A zero membership for ladar indicates the presence of an obstacle in the path and for vision, the end of the row. The variable $x$ takes the value of 0 to $1 \mathrm{~m}$ for zero, 1 to $3 \mathrm{~m}$ for reasonable, and any value greater than $2 \mathrm{~m}$ for unreasonable. The ranges were intuitively chosen to be the possible values. Normalization of the linguistic variables was performed at a scale from 0 to 1 . During the presence of an obstacle, the ladar takes precedence until the obstacle is avoided. The rule set for the supervisor is shown in table 1 . Each rule received a corresponding normalized membership value from the input membership function $x$. A rule-based inference engine was used to make the inferences. Rule sets were extracted using the rule matrix shown in table 1 . Based on the linguistic variables chosen from each $x$, the corresponding rules are fired. From the rules fired for a given instant, the output membership function is chosen. The membership value was used to find the crisp value for the output. The output membership function decision is shown in figure $4 \mathrm{~b}$, which relates the rules fired to the linguistic variables vision, ladar, and both. These variables represent the decision made as to whether vision or ladar is given higher priority or if both should be given equal priority. Defuzzification was done using the center of gravity method. The crisp value of the decision is taken as the measurement noise covariance value for that sensor. For example, if a decision of ladar is obtained with a crisp value of 1 , then $\sigma_{X l}$ is updated to 1 and $\sigma_{X C}$ is chosen as 0 . If a decision is made as both with a crisp value 


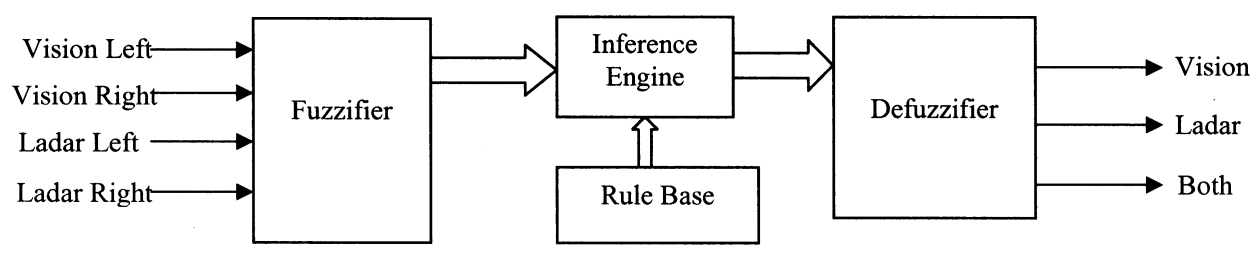

(a)



(b)

Figure 4. (a) Fuzzy logic structure and (b) membership functions for sensor supervisor.

Table 1. Fuzzy logic rule set for sensor supervisor.

\begin{tabular}{ccc}
\hline Vision Left/Right & Ladar Left/Right & Decision \\
\hline Reasonable & Reasonable & Both \\
& Reasonable/zero & Ladar higher \\
& Zero & Stop \\
& Unreasonable & Vision \\
& Unreasonable/zero & Ladar \\
& Reasonable/unreasonable & Vision higher \\
\hline Unreasonable & Reasonable & Ladar \\
Reasonable/zero & & Ladar higher \\
Zero & & Ladar \\
Unreasonable/zero & & Ladar \\
Reasonable/unreasonable & & Both \\
\hline
\end{tabular}

of -0.5 , then $\sigma_{X c}$ is chosen as 0.5 and $\sigma_{X l}$ is chosen as $(-0.5 /-1) \times 0.2=0.1$. The positive or negative values only indicate whether the decision is vision or ladar. Only the absolute values are used to update $\boldsymbol{R}$. The range of crisp values for the decision was chosen by performing simulations and determining which values provide good results. The details of the simulation are provided later in the Simulation section.

\section{Divergence Detection and Fuzzy Logic CoRrection}

The innovation vector $z^{\prime}(k)$ (Klein, 1999) is defined as the difference between the measured vector and the estimation of the measurement vector:

$$
z^{\prime}(k)=\boldsymbol{z}(k)-\boldsymbol{H} \boldsymbol{x}(k)
$$

For an optimum Kalman filter, an innovation vector of zero mean Gaussian white noise (Abdelnour et al., 1993). Therefore, the performance of the Kalman filter can be monitored using the value of the innovation vector. Deviation of $z$ ' from zero by more than $5 \%$ was taken as the indication

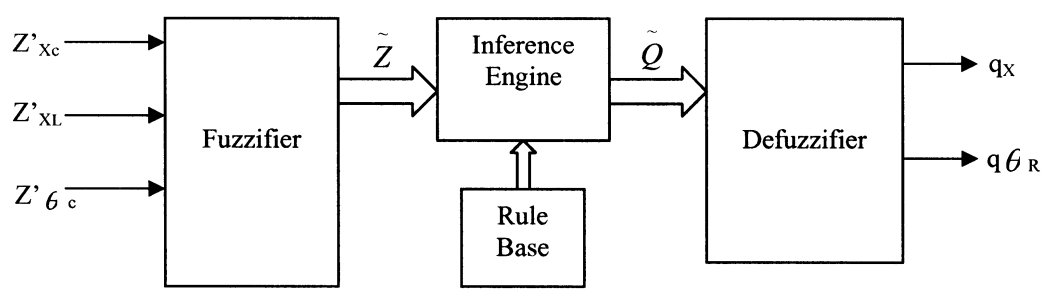

(a)
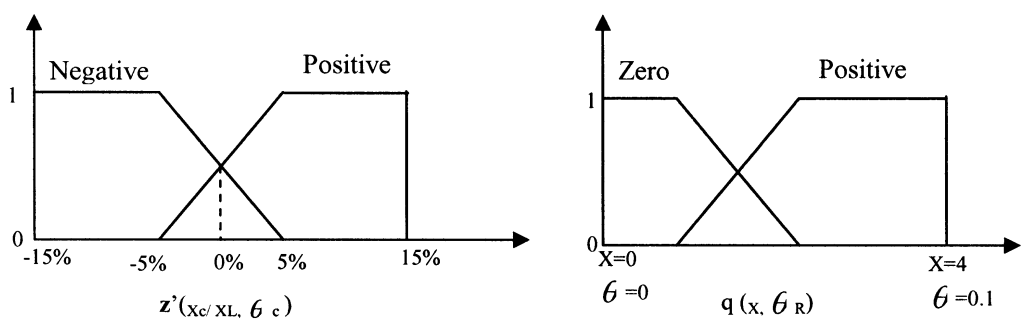

(b)

Figure 5. (a) Fuzzy logic structure and (b) membership functions for updating $Q$. 
Table 2. Fuzzy logic rule set for divergence correction.

\begin{tabular}{cccc}
\hline$z_{X}^{\prime}$ & $z^{\prime} \theta_{c}$ & $q_{X}$ & $q \theta_{R}$ \\
\hline Negative & Negative & Zero & Zero \\
Negative & Positive & Zero & Positive \\
Positive & Negative & Positive & Zero \\
Positive & Positive & Positive & Positive \\
\hline
\end{tabular}

of reduction in performance of the filter leading towards divergence.

Divergence could be corrected by updating the process noise covariance matrix $\boldsymbol{Q}$, depending on the innovation sequence. Fuzzy logic (Passino and Yurkovich, 1998; Subramanian et al., 2005) was used to perform this for two parameters, $q_{X}$ and $q \theta_{R}$. The fuzzy logic architecture and membership functions are shown in figure 5 .

The $x_{c}, x_{L}$, and $\theta_{c}$ parameters of the innovation vector $z^{\prime}$ are the input to the fuzzy logic system. The ranges of the input are from $-15 \%$ to $15 \%$. These values were chosen by trial and error by performing simulation. Normalization of the linguistic variables was performed at a scale from 0 to 1 . Based on the above argument for correction, a set of if-then rules was developed by experimentation. Each rule received a corresponding normalized membership value from the input membership function $z$ '. Based on the linguistic variables chosen from each $z$, the corresponding rules are fired. The rule set is shown in table 2 . For example, consider the second row in the table, where $z_{X}^{\prime}$ is negative and $z^{\prime} \theta_{C}$ is positive. This indicates that the error in position has decreased compared to the previous time step, but the vehicle is heading in the wrong direction. The process noise for position, $q_{X}$, is zero, and the process noise for heading, $q \theta_{R}$, is positive. In the rule set, a single measurement value for position, $z_{X}{ }_{X}$, is given instead of the two different values, $z_{X C}$ and $z_{X L}^{\prime}$. This is because the sensor supervisor described earlier picks one of the sensors for use in the filter when the other is unreasonable. When one sensor is given higher priority, the position measurement of that sensor alone is used in the rule set; when both sensors are selected by the supervisor, the position measurement of ladar alone is used, as it has lower measurement noise. This was done to reduce the complexity of the rule set.

From the rules fired for a given instant, the output membership function was chosen. The membership value was used to find the crisp value for the output. Defuzzification was done using the center of gravity method (Passino and Yurkovich, 1998). The crisp values of the process noise covariance are then updated in the process noise covariance matrix $\boldsymbol{Q}$. The range of crisp values was chosen by trial and error by performing simulations and determining which values provide good results.

For the parameters, $q \theta_{C}$ and $q_{V}$, a linear relationship was followed such that $q \theta_{C}$ and $q_{V}$ were increased proportional to $\left|z^{\prime}-z\right|$ of $\theta_{C}$ and $v$, respectively. The speed of the vehicle obtained from the speed sensor was used to scale the output to the steering control. The implementation of the steering mechanism and control was described by Subramanian et al. (2006). From the past research conducted on this vehicle, it was observed that for a given steering angle, higher speed resulted in larger lateral displacement. The transfer function between the required lateral displacement of the vehicle to reduce the error to zero, as determined from the fusion algorithm, and the steering angle is given by equation 8 , and the gains were determined experimentally for speeds of 1.8, 3.1, and $4.4 \mathrm{~m} \mathrm{~s}^{-1}$ (Subramanian et al., 2006):

$$
G(s)=\frac{k}{s^{2}}
$$

To determine the steering angle at different speeds, the steering angle at $4.4 \mathrm{~m} \mathrm{~s}^{-1}$ was scaled using equation 9 . The scaling of the gain, $G(s)$, for $4.4 \mathrm{~m} \mathrm{~s}^{-1}$ produced the least error at different speeds compared to the gains for 1.8 and $3.1 \mathrm{~m}$ $\mathrm{s}^{-1}$ :

Steering angle $=$ gain $\times$ steering angle at $4.4 \mathrm{~m} \mathrm{~s}^{-1}$

where gain $=\left(\right.$ current speed $\left./ 4.4 \mathrm{~m} \mathrm{~s}^{-1}\right)$.

\section{Simulation}

The developed fuzzy-Kalman filter algorithm was simulated in MATLAB (version 7.0) to check the functionality and accuracy. The code used for simulation was custom written in MATLAB. The input data required for simulation were obtained by manually driving the vehicle through a citrus grove alleyway and collecting all the sensor information. The input data were the values measured by the sensors, the time between measurements, and errors from the machine vision and ladar algorithms. The data from runs on

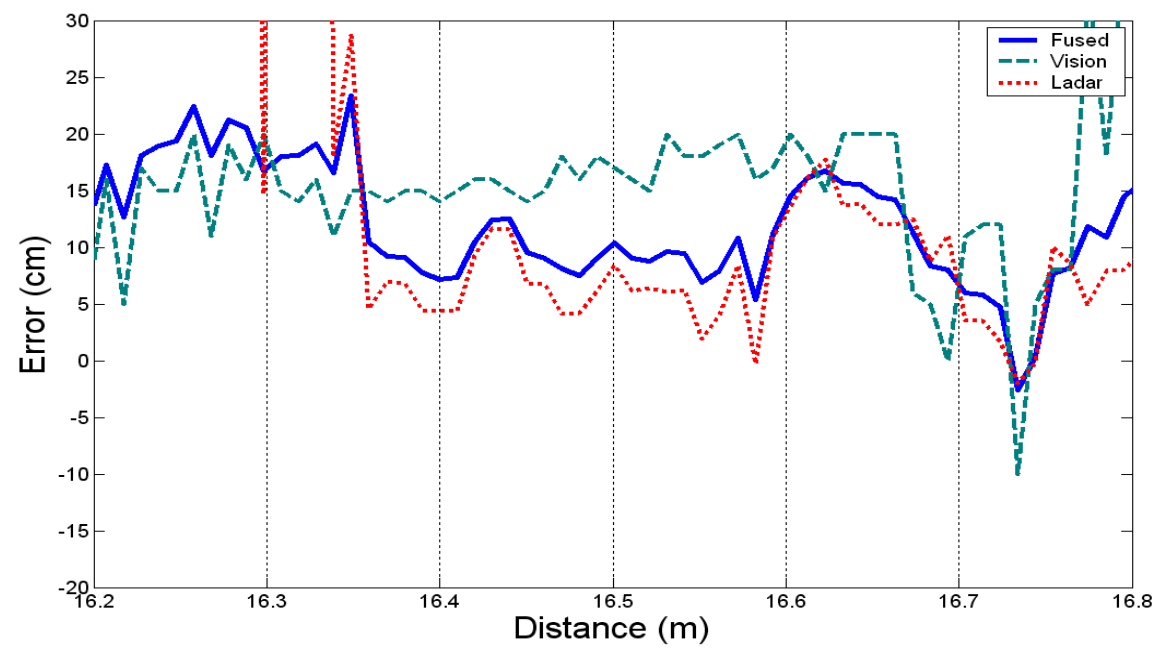

Figure 6. Simulation result of fusion of error obtained from vision and ladar algorithms. 
three different grove alleyways were used for three different simulation runs. These data were from approximately $360 \mathrm{~s}$ of vehicle run time. The output is the resultant error obtained after passing the input data to the fusion algorithm. The aim of the simulation was to check that the algorithm was operating as required, i.e., the suitable sensor is chosen by the sensor supervisor, divergence does not occur, and large variations reported by the sensors are dampened.

Figure 6 shows the simulated result of fusing the error obtained from the vision and ladar algorithms. The figure shows both the input and output data. The error from vision and ladar are the input (dashed lines). The output is the error after fusion (solid line). The error is the distance between the center of the vehicle in the path and the path center. For clarity, the result is shown for a section of the path from 16.2 to $16.8 \mathrm{~m}$ of travel. It is observed that from 16.2 to $16.35 \mathrm{~m}$, only vision provides reasonable error and ladar does not. This may be attributed to the fact that ladar sweep at that location might have been in a gap between two consecutive trees. Hence, for the first $15 \mathrm{~cm}$ distance, the fused data seems to have relied mainly on vision. For the 16.35 to $16.55 \mathrm{~m}$ section, errors from both vision and ladar algorithms seem reasonable; hence, fusion takes both data into account. However, the fused data rely more on ladar information, as the process noise is less for ladar. For the last $25 \mathrm{~cm}$ of the section from 16.55 to $16.8 \mathrm{~m}$, both vision and ladar determine the same amount of error. The simulation results verify that the aim of the simulation was met.

\section{Experimental Procedure}

Because citrus grove alleyways are typically straight rows, it was felt that testing the vehicle on curved paths at different speeds would be a more rigorous test of the guidance system's performance than directly testing in straight grove alleyways. This test would represent the ability of the guidance system to navigate more difficult conditions than the ones likely to be encountered in the grove. For this purpose, a flexible wall track was constructed using hay bales to form an S-shaped test path. This shape provided both straight and curved conditions. The path width varied from 3 to $4.5 \mathrm{~m}$, and the total path length was approximately $53 \mathrm{~m}$. A gap of approximately $1 \mathrm{~m}$ was left between successive hay bales. The gaps mimicked missing trees in a citrus grove alleyway. The profile of the hay bale track is shown in figure 7. The hay bales provided a physically measurable barrier, which aided in operating the ladar guidance system, and color contrast with grass, which is useful for vision based guidance. In addition, when testing a large tractor, only minor

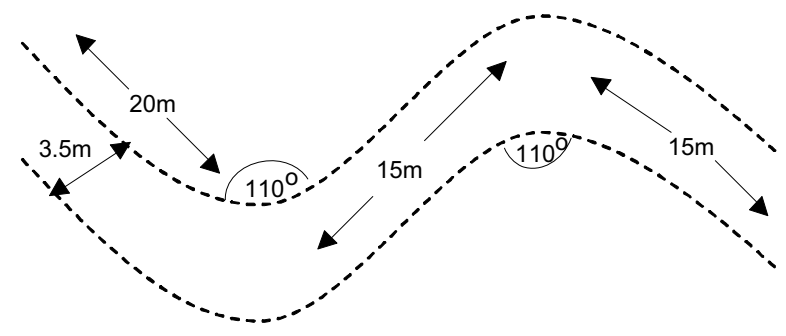

(a)

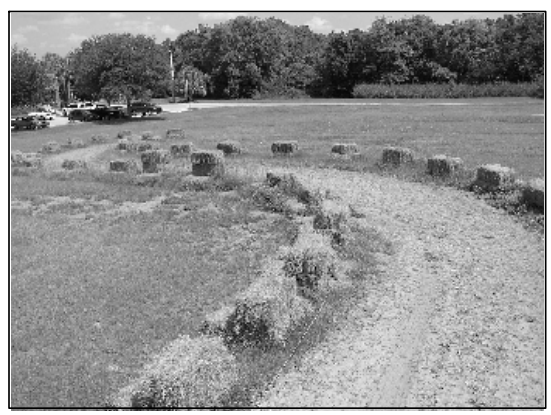

(b)

Figure 7. Hay bale track profile: (a) dimensions and (b) photo.

losses would occur if the tractor were to run over a barrier of hay bales.

Experiments were conducted to check the robustness and accuracy of the fused guidance system in the test path at 1.8 and $3.1 \mathrm{~m} \mathrm{~s}^{-1}$. The vehicle was manually navigated to the starting position at one end of the test path. Once positioned, the vehicle was started in autonomous control and allowed to navigate down the path to the other end, thereby navigating a distance of $53 \mathrm{~m}$. Three repetitions were performed for each vehicle speed to ensure replication, resulting in a total of six trial runs. The path traveled was marked on the soil by a marking wheel attached to the rear of the tractor. The error measurements were taken manually using a tape measure at regular intervals of $1 \mathrm{~m}$. This interval was chosen to get enough samples to correctly estimate the path error. Measurements at smaller intervals were redundant.

After achieving satisfactory performance, wherein the vehicle stayed close to the center of the test path without hitting the hay bales, tests were conducted in orange grove alleyways at the Pine Acres experimental station of the University of Florida. The average alleyway path width was approximately $3.5 \mathrm{~m}$, and the length of the alleyway was approximately $110 \mathrm{~m}$ with about 30 trees on either side. A
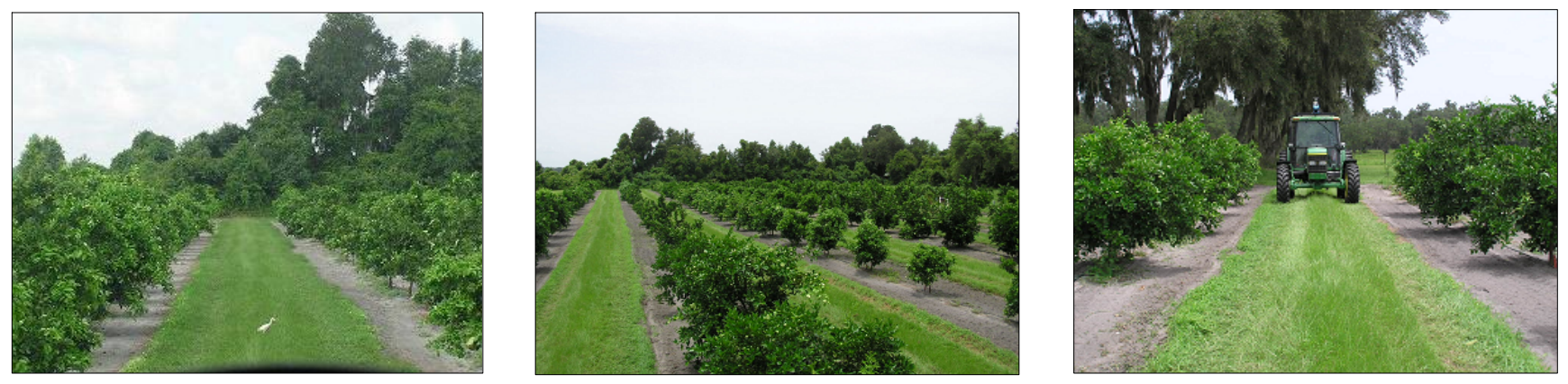

Figure 8. Citrus grove alleyways. 


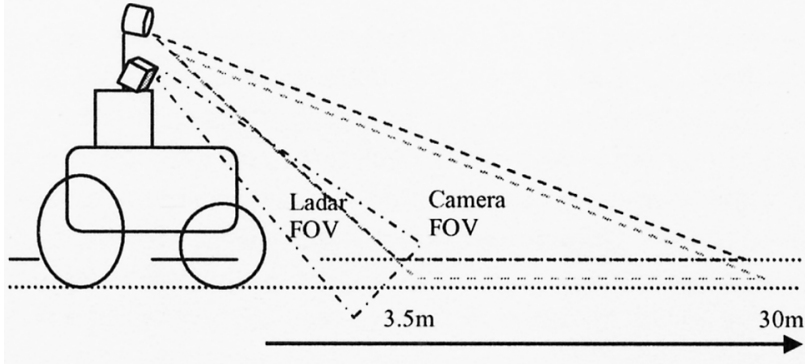

(a)

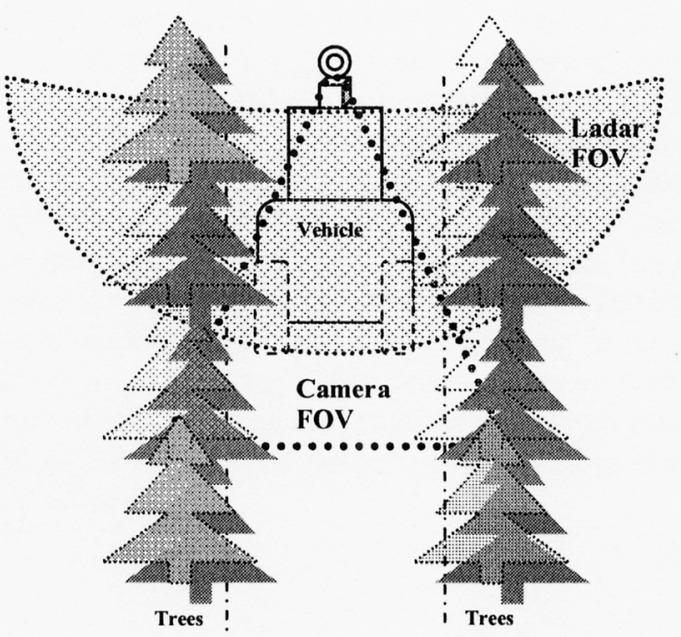

(b)

Figure 9. Field of view (FOV) of camera and ladar: (a) side view and (b) top/front view when the vehicle is located in a grove alleyway.

typical alleyway is shown in figure 8 , where the approximate tree height is 1.5 to $2 \mathrm{~m}$. Figure 9 shows the field of view of the camera and the ladar when navigating the citrus grove alleyway.

Experiments were conducted at speeds of 1.8 and $3.1 \mathrm{~m} \mathrm{~s}^{-1}$ along an alleyway. Three repetitions were conducted for each speed. For measuring the error, markers were laid on the ground at the path center at approximately $4 \mathrm{~m}$ intervals along the alleyway. To locate each marker, the path center was determined as the center point between tree edges on either side of the alleyway and measured manually using a tape measure. The test path with harder turns required a sampling interval of $1 \mathrm{~m}$, whereas the grove alleyway was a relatively straight path and therefore a sampling interval of $4 \mathrm{~m}$ was sufficient. A rear-facing camera was mounted on top of the tractor cab at the center of the vehicles' width. The camera's field of view included the area of ground just behind the rear of the tractor. Pixel to horizontal distance information was calibrated by correlating the field of view of the camera with the corresponding real-world distance. While conducting the experiments, video of the ground behind the tractor was collected using this camera. The error in traversing the alleyway was determined based on the position of the markers at different instances in the video. The boundary of the path for determining the path center in both the test track and in the grove was delimited manually by visual approximation. The method employed in the test track of marking the path on the ground followed by measuring with a tape measure was not a viable option, as the marking instrument did not provide good marks on the ground in the alleyway. Further, manual measurement using a tape measure for a long path such as a grove alleyway was labor intensive. It was recognized that the pitch and roll of the vehicle would affect the measurements made using this video-based measurement, but the effect was assumed to be negligible compared to the width of the path.

\section{RESUlts AND DiscusSION}

From the experiments, RMS error, average error, maximum error, and standard deviation of error were calculated over the entire path by averaging the data over the three repetitions at each speed. The path error, which was the deviation from the center line of the alleyway, was plotted over the length of the test path for the different speeds. The positive and negative values of the error in the plot are only an indication of the error being on the right side or left side of the path center. For calculating the performance measures, the absolute values of the error were used. Performance measures obtained from the experiments conducted in the test track are shown in table 3 , and the corresponding path error is shown in figure 10. In figure 10, actual points collected are shown as solid dots, and dotted lines indicate the vehicle's path. Visually, the vehicle navigated in the middle of the path as well as a human driver would. The average error in both cases was less than $2 \mathrm{~cm}$ from the path center. The measures were lower for the lower speed of $1.8 \mathrm{~m} \mathrm{~s}^{-1}$ than at $3.1 \mathrm{~m} \mathrm{~s}^{-1}$. The maximum error at both speeds was less than $4 \mathrm{~cm}$ in the path width of at least $3 \mathrm{~m}$. This was an error of only $1 \%$ of the path width. From figure $10 \mathrm{a}$, it can be observed that the error was less than $2 \mathrm{~cm}$ most of the time, and only in a few instances did the maximum error exceed $2 \mathrm{~cm}$.

The results of running vision and ladar based guidance separately on similar but not identical test tracks were reported by Subramanian et al. (2006). However, the path used in the present research was more complex. At speeds of $3.1 \mathrm{~m} \mathrm{~s}^{-1}$, an average error of $2.5 \mathrm{~cm}$ was reported while using ladar based guidance, whereas guidance based on fusion had an average error of $1.9 \mathrm{~cm}$ at the same speed. The maximum error was also reduced from $5 \mathrm{~cm}$ reported for vision based guidance at $3.1 \mathrm{~m} \mathrm{~s}^{-1}$ to $4 \mathrm{~cm}$ using fusion based guidance. Compared to those methods of individual sensor guidance, navigation using sensor fusion resulted in better performance in staying closer to the path center. It should be noted that the tests of individual vision and ladar based sensors were conducted on similar but different test tracks as compared with the fusion based tests. Although the improvements in using fusion may not be statistically significant from using individual sensor based guidance on test tracks, a significant benefit of using fusion was observed in grove conditions. For example, in cases where a tree is missing in the alleyway, ladar based guidance is expected to provide bad results while navigating close to headlands, and vision based guidance is expected to perform poorly due to the limited field of view of the mount configuration, as described earlier in the Approach section. Therefore, the significant advantage of fusion is in versatility and reliability rather than in accuracy.

As directly observed from the simulations, the reduction of noise also played a significant role in the improvement of 


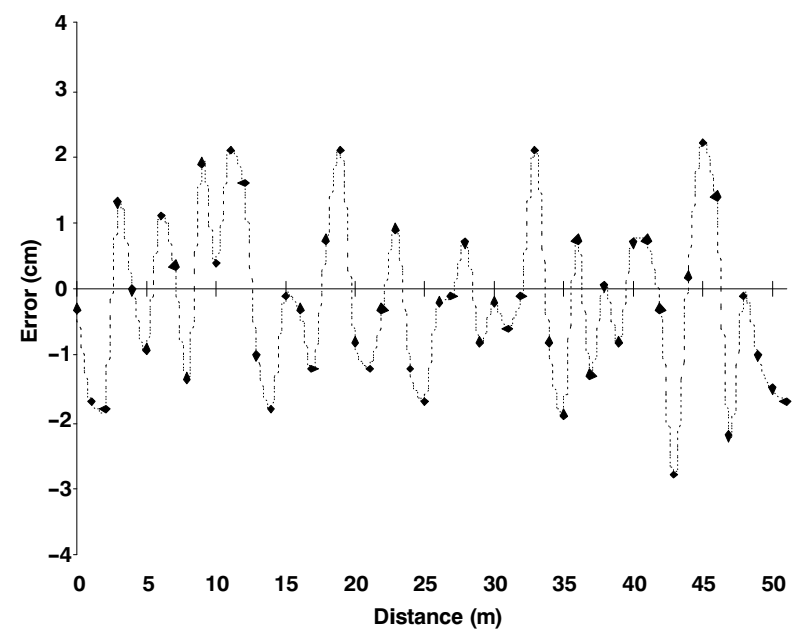

(a)

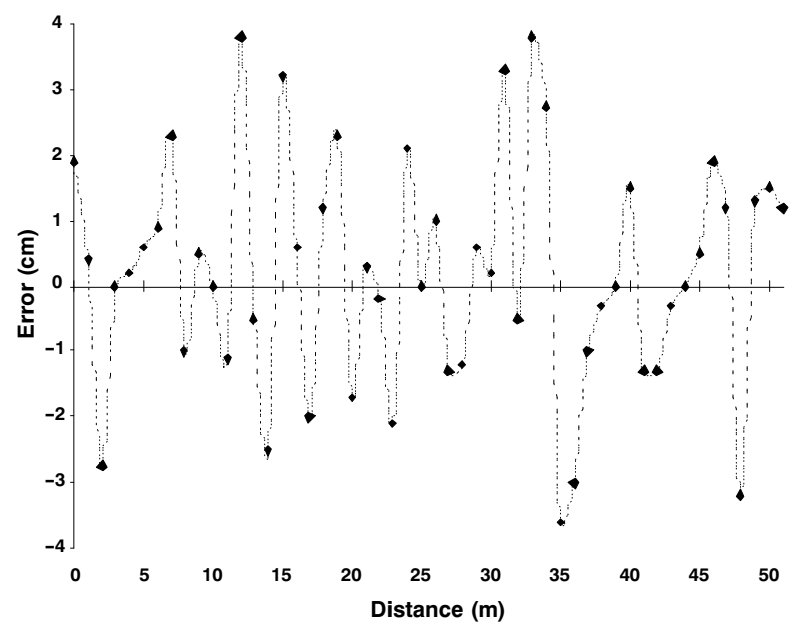

(b)

Figure 10. Path navigation error of the vehicle in the test track at (a) $1.8 \mathrm{~m}$ $\mathrm{s}^{-1}$ and (b) $3.1 \mathrm{~m} \mathrm{~s}^{-1}$.

performance. This was in agreement with results reported by Han et al. (2002) that use of a Kalman filter reduced the noise and improved the tracking performance for DGPS based parallel tracking. Kobayashi et al. (1998) reported high accuracy by using a fuzzy logic Kalman filter based fusion method using DGPS, in accordance with the results reported in this article. Sasladek and Wong (1999) used fuzzy logic for tuning the Kalman filter with DGPS as the primary sensor and reported that the simulation results showed prevention of divergence and improved accuracy in position and velocity. Comparatively, this research used vision and ladar as sensors and prevented divergence using fuzzy logic for tuning. In addition, fuzzy logic was also used for picking the more accurate sensor, and improved performance was

Table 3. Performance measures obtained from the experiments conducted in test track.

\begin{tabular}{ccccc}
\hline $\begin{array}{c}\text { Speed } \\
\left(\mathrm{m} \mathrm{s}^{1}\right)\end{array}$ & $\begin{array}{c}\text { Average } \\
\text { Error } \\
(\mathrm{cm})\end{array}$ & $\begin{array}{c}\text { Standard } \\
\text { Deviation } \\
(\mathrm{cm})\end{array}$ & $\begin{array}{c}\text { Maximum } \\
\text { Error } \\
(\mathrm{cm})\end{array}$ & $\begin{array}{c}\text { RMS } \\
\text { Error } \\
(\mathrm{cm})\end{array}$ \\
\hline 1.8 & 1.5 & 0.7 & 3 & 1.6 \\
3.1 & 1.9 & 1 & 4 & 2.1 \\
\hline
\end{tabular}

experimentally verified for a citrus grove application. This research demonstrates significant improvements and differences compared to previous research on autonomous vehicles for agricultural applications. Whereas DGPS has been the primary guidance sensor for many research studies reported in the literature, this research uses machine vision and ladar as the primary sensors for guidance. Secondly, most previous research used fuzzy logic exclusively for tuning or divergence detection. This research used fuzzy logic for tuning or divergence correction as well as for adaptively choosing which sensor was to be relied upon for fusion at different locations in the path. This method allowed for switching between important sensors that are useful in specific areas. Further, previous research in agriculture mainly focused on crop applications, whereas this research focuses on citrus grove applications.

Performance measures obtained from the experiments conducted in the citrus grove alleyway are shown in table 4, and the error plot is shown in figure 11. In figure 11, actual points collected are shown as solid dots, and dotted lines indicate the vehicle's path. The average error at the two speeds was less than $10 \mathrm{~cm}$, and the maximum error was less than $22 \mathrm{~cm}$. This was about $6 \%$ of the path width. The standard deviation of error was $4.1 \mathrm{~cm}$ for $1.8 \mathrm{~m} \mathrm{~s}^{-1}$ and $4.4 \mathrm{~cm}$ for $3.1 \mathrm{~m} \mathrm{~s}^{-1}$. The standard deviation was larger compared to the standard deviation observed in the test path. The root mean squared (RMS) error was $8.6 \mathrm{~cm}$ at $1.8 \mathrm{~m} \mathrm{~s}^{-1}$ and $10.3 \mathrm{~cm}$ at $3.1 \mathrm{~m} \mathrm{~s}^{-1}$. Tsubota et al. (2004) reported an RMS error of $10 \mathrm{~cm}$ in navigating an orchard at a speed of $1 \mathrm{~m}$ $\mathrm{s}^{-1}$ using an all-terrain vehicle. In comparison, this research used a tractor, which was a much larger vehicle and achieved an accuracy of $8.6 \mathrm{~cm}$ RMS error at a speed of $1.8 \mathrm{~m} \mathrm{~s}^{-1}$. This shows the better performance of fusion based guidance in navigating an alleyway. Moreover, Tsubota et al. (2004) reported mounting the ladar at a low level on the vehicle such that scanning only detected the trunk of the trees. Such a method does not take into account the variation in canopy, whereas the method used in this research accounted for the variation in tree canopy, and navigation was based on the canopy variation. Cho and $\mathrm{Ki}$ (1999) used machine vision and ultrasonic sensor with fuzzy logic control to steer a sprayer through an orchard. They reported an error of $25.29 \mathrm{~cm}$ (RMS) using machine vision. The error of $10.3 \mathrm{~cm}$ (RMS) at $3.1 \mathrm{~m} \mathrm{~s}^{-1}$ observed in this research was much lower. These results emphasize the role of fusion based guidance in navigating through orchards.

From the error plots, it seems like there are error spikes at a few locations. However, it should be noted that the distance over which the error plot is made was more than $100 \mathrm{~m}$. Therefore, the errors vary over a large distance and should not be seen as spikes. It should also be observed that there was variation in the lateral position of the vehicle at the starting location of the alleyway. This is shown from the initial errors observed in the plots. From the error plot, it can be observed that the error was less than $15 \mathrm{~cm}$ most of the time. Based on

Table 4. Performance measures obtained from the experiments conducted in grove alleyway.

\begin{tabular}{ccccc}
\hline $\begin{array}{c}\text { Speed } \\
\left(\mathrm{m} \mathrm{s}^{1}\right)\end{array}$ & $\begin{array}{c}\text { Average } \\
\text { Error } \\
(\mathrm{cm})\end{array}$ & $\begin{array}{c}\text { Standard } \\
\text { Deviation } \\
(\mathrm{cm})\end{array}$ & $\begin{array}{c}\text { Maximum } \\
\text { Error } \\
(\mathrm{cm})\end{array}$ & $\begin{array}{c}\text { RMS } \\
\text { Error } \\
(\mathrm{cm})\end{array}$ \\
\hline 1.8 & 7.6 & 4.1 & 18 & 8.6 \\
3.1 & 9.4 & 4.4 & 22 & 10.3 \\
\hline
\end{tabular}




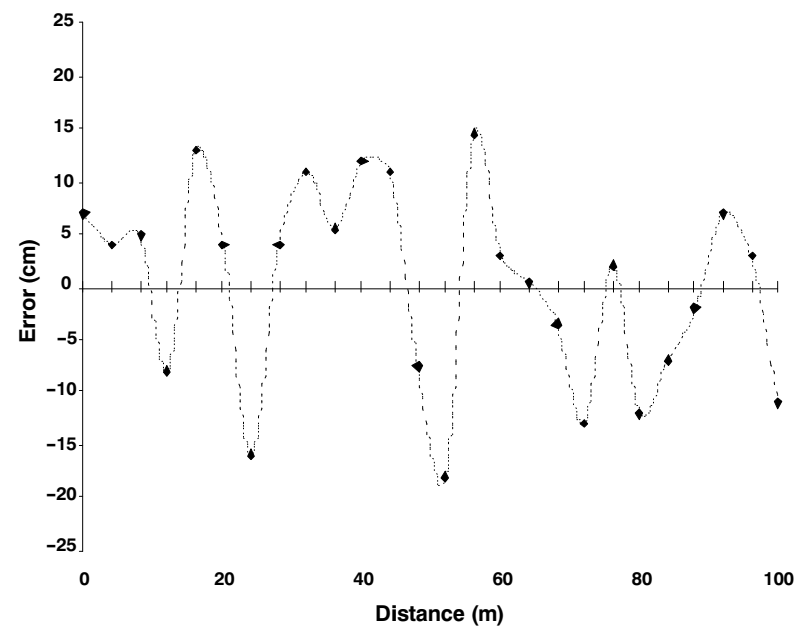

(a)



(b)

Figure 11. Path navigation error of the vehicle in the grove alleyway at (a) $1.8 \mathrm{~m} \mathrm{~s}^{-1}$ and (b) $3.1 \mathrm{~m} \mathrm{~s}^{-1}$.

the performance measures, the guidance seems less accurate in the relatively straight grove than in the complex test path. However, it should be noted that the path boundary conditions in the grove are much more variable than in the test path. In the test track, the path boundary was relatively even without much variation in the location between successive hay bales. In the citrus grove, the tree canopy size varied significantly from one tree to the next, from as low as $5 \mathrm{~cm}$ to as high as $50 \mathrm{~cm}$. The path boundary used for error measurement, which was the tree canopy edge, was delimited by the person conducting the experiment, and it was not practical to accurately quantify a tree canopy boundary. The larger errors in the citrus grove can be largely attributed to this boundary variability rather than the navigation performance of the vehicle. These high errors were still acceptable to navigate sufficiently in the middle of the alleyway without hitting any tree branches. Visually, the navigation in the alleyway was as good as human driving and as good as navigation in the test track. Overall, assuming a human driver as a reference of performance, navigation in the citrus grove was as good as human driving with reasonable errors and confirms the good performance of fusion based guidance.

\section{Conclusion}

A sensor fusion system using a fuzzy logic enhanced Kalman filter was designed to fuse the information from machine vision, ladar, IMU, and speedometer. Simulations were performed to confirm the operation of the method. The method was then implemented on a commercial tractor. The fusion based guidance system was first tested on customdesigned test tracks. Tests were conducted for two different speeds of 1.8 and $3.1 \mathrm{~m} \mathrm{~s}^{-1}$. Average errors of $1.9 \mathrm{~cm}$ at $3.1 \mathrm{~m}$ $\mathrm{s}^{-1}$ and $1.5 \mathrm{~cm}$ at $1.8 \mathrm{~m} \mathrm{~s}^{-1}$ were observed in the tests. The maximum error was not more than $4 \mathrm{~cm}$ at both speeds. Tests were then conducted at the same speeds in a citrus grove alleyway. The average error was less than $10 \mathrm{~cm}$ for both speeds. The guidance system's ability to navigate was verified in citrus grove alleyways, and the system was found to perform well, with the vehicle remaining close to the center of the alleyway at all times. The developed fusion based guidance system was found to be more accurate than individual sensor based guidance systems on test paths. The fusion based guidance system enables accurate navigation in alleyways with missing trees and end-of-alleyway navigation, where individual sensor based guidance cannot be relied upon. The present system is able to accurately traverse an alleyway of a citrus grove.

Additional work is necessary before the system can be ready for production. Some of the future work includes adding the ability to navigate the headlands and the ability to optimally navigate the entire grove. Future tests should also consider other vehicle speeds.

\section{ACKNOWLEDGEMENTS}

Research conducted at the University of Florida, Institute of Food and Agricultural Sciences. Special thanks are extended to M. Zingaro and G. Pugh for their support.

\section{REFERENCES}

Abdelnour, G., S. Chand, and S. Chiu. 1993. Applying fuzzy logic to the Kalman filter divergence problem. In Proc. Intl. Conf. on Systems, Man and Cybernetics 1: 630-635. Piscataway, N.J.: IEEE.

Chen, B., S. Tojo, and K. Watanabe. 2003. Machine vision based guidance system for automatic rice planters. Applied Eng. in Agric. 19(1): 91-97.

Cho, S. I., and N. H. Ki. 1999. Autonomous speed sprayer guidance using machine vision and fuzzy logic. Trans. ASAE 42(4): $1137-1143$

Davis, I. L., and A. Stentz. 1995. Sensor fusion for autonomous outdoor navigation using neural networks. In Proc. IEEE/RSJ Intl. Conf. on Intelligent Robots and Systems 3: 338-343. Piscataway, N.J.: IEEE.

Fitzgerald, R. 1971. Divergence of the Kalman filter. IEEE Trans. Automatic Control 16(6): 736-747.

Gage, A., and R. R. Murphy. 2000. Sensor allocation for behavioral sensor fusion using min-conflict with happiness. In Proc. IEEE/RSJ Intl. Conf. on Intelligent Robots and Systems 3: 1611-1618. Piscataway, N.J.: IEEE.

Han, S., Q. Zhang, and H. Noh. 2002. Kalman filtering of DGPS positions for a parallel tracking application. Trans. ASAE 45(3): 553-559. 
Klein, L. A. ed. 1999. Sensor and data fusion concepts and applications. Bellingham, Wash.: SPIE.

Kobayashi, K., K. C. Cheok, K. Watanabe, and F. Munekata. 1998. Accurate differential global positioning system via fuzzy logic Kalman filter sensor fusion technique. IEEE Trans. Ind. Electronics 45(3): 510-518.

Mobus, R., and U. Kolbe. 2004. Multi-target multi-object tracking, sensor fusion of radar and infrared. In Proc. IEEE Intelligent Vehicles Symp., 732-737. Piscataway, N.J.: IEEE.

Nagasaka, Y., N. Umeda, Y. Kanetai, K. Tanikawi, and Y. Sasaki. 2004. Automated rice transplanter using global positioning and gyroscopes. Computers and Electronics in Agric. 43(3): 223-234.

Noguchi, N., M. Kise, K. Ishii, and H. Terao. 2002. Field automation using robot tractor. In Proc. Automation Tech. for Off-Road Equipment, 239-245. St. Joseph, Mich.: ASABE.

Passino, K. M, and S. Yurkvovich. 1998. Fuzzy Control. Menlo Park, Cal.: Addison Wesley Longman.

Paul, A. S., and E. A. Wan. 2005. Dual Kalman filters for autonomous terrain aided navigation in unknown environments. In Proc. IEEE Intl. Joint Conf. on Neural Networks, 5: 2784-2789. Piscataway, N.J.: IEEE.

Rasmussen, C. 2002. Combining laser range, color, and texture cues for autonomous road following. In Proc. IEEE Intl. Conf. on Robotics and Automation 4: 4320-4325. Piscataway, N.J.: IEEE. Runkler, T., M. Sturm, and H. Hellendoorn. 1998. Model-based sensor fusion with fuzzy clustering. In Proc. IEEE Intl. Conf. on Fuzzy Systems 2: 1377-1382. Piscataway, N.J.: IEEE.
Sasladek, J. Z, and Q. Wang. 1999. Sensor fusion based on fuzzy Kalman filtering for autonomous robot vehicle. In Proc. IEEE Intl. Conf. on Robotics and Automation 4: 2970-2975. Piscataway, N.J.: IEEE.

Satow, T., K. Matsuda, S. B. Ming, K. Hironaka, and D. L. S. Tan. 2004. Development of laser crop row sensor for automatic guidance system of implements. In Proc. Automation Tech. for Off-Road Equipment, 131-139. St. Joseph, Mich.: ASABE.

Subramanian, V., T. F. Burks, and S. Singh. 2005. Autonomous greenhouse sprayer vehicle using machine vision and laser radar for steering control. Applied Eng. in Agric. 21(5): 935-943.

Subramanian, V., T. F. Burks, and A. A. Arroyo. 2006. Development of machine vision and laser radar based autonomous vehicle guidance systems for citrus grove navigation. Computers and Electronics in Agric. 53(2): 130-143.

Tsubota, R., N. Noguchi, and A. Mizushima, 2004. Automatic guidance with a laser scanner for a robot tractor in an orchard. In Proc. Automation Tech. for Off-Road Equipment, 369-373. St. Joseph, Mich.: ASABE.

Wu, H., M. Siegel, R. Stiefelhagen, and J. Yang. 2002. Sensor fusion using Dempster-Schafer theory. In Proc. IEEE Instrumentation and Measurement Conf. 1: 7-12. Piscataway, N.J.: IEEE.

Zarchan, P., and H. Musoff, eds. 2005. Fundamentals of Kalman Filtering: A Practical Approach. Progress in Astronautics and Aeronautics, volume 208. Reston, Va.: AIAA. 